\title{
The safety and efficacy of SDLT-2 (Sodium-glucose co-transporter 2) inhibitor versus placebo in the treatment of heart failure in patients with or without T2DM (Type 2 diabetes mellitus): A Systemic Review and Meta-analysis.
}

Qian Zhang

Yantaishan Hospital

Xiaofei Wang

Yantaiyuhuangding Hospital

Peipei Ge

Yantaiyuhuangding Hospital

Aizhen Hu

Yantaiyuhuangding Hospital

Xuexun Li ( $\nabla$ lixuexun2005@163.com )

Yantaiyuhuangding Hospital https://orcid.org/0000-0002-6554-7010

Original investigation

Keywords: SGLT2 inhibitor, heart failure, meta-analysis

Posted Date: March 11th, 2020

DOl: https://doi.org/10.21203/rs.3.rs-16652/v1

License: (c) (1) This work is licensed under a Creative Commons Attribution 4.0 International License.

Read Full License 


\section{Abstract}

\section{Background}

Sodium-glucose co-transporter 2 (SGLT2) inhibitor which is a type of drug used for the treatment of diabetes mellitus, has been reported by many trials that it could be beneficial for patients with established heart failure. A meta-analysis on this subject could obtain more reliable estimates of the efficacy and safety outcomes.

\section{Methods}

A systematic review and meta-analysis of randomized, placebo-controlled trials of SGLT2 inhibitor in patients with heart failure was conducted. We searched PubMed, Cochrane Library, and Web of Science for trials published from inception to March, 2018. PRISMA guidelines (Preferred Reporting Items for Systematic Reviews and Meta-Analyses) was used to conduct the review. For quality assessment of included studies. The methodological quality of the included trials was assessed using the Cochrane tool for assessing randomized clinical trials (RCT). Efficacy outcomes included hospitalization for heart failure and all-cause death. Safety outcomes consisted of serious adverse event (SAE) and volume depletion.

\section{Results}

We included data from 5 identified studies and 8775 patients (aged 64.9, female 29.8\%). A total of 3930 (44.8\%) patients were known to have diabetes mellitus. Compared with placebo, SGLT2 inhibitor decrease the incidence of hospitalization for heart failure (RR 0.692; $95 \% \mathrm{Cl}, 0.611-0.784 \mathrm{P}<0.001)$, and all-cause death $(\mathrm{RR} 0.824 ; 95 \% \mathrm{Cl}, 0.736-0.922 \mathrm{P}=0.001)$. The incidence of $\mathrm{SAE}$ in patients with a treatment of SGLT2 inhibitor was low (RR 0.869; $95 \% \mathrm{Cl}, 0.779-0.970 \mathrm{P}=0.012)$. SGLT2 inhibitor didn't increase the incidence of volume depletion (RR1.165, 95\%Cl, 0.977-1.390 P=0.089).

\section{Conclusion}

Our results confirm that SGLT2 inhibitor is effective and safe for patients with heart failure regardless of presence of diabetes mellitus.

\section{Background}

Heart failure is a main cause for hospitalization annually [1]. Patients with heart failure have a high financial burden, and are at high risk for rehospitalization and death [2]. Despite advances in medicine and new therapeutic devices, outcomes for patients with heart failure are still poor[3]. Many medical interventions were demonstrated to reduce the incidence of mortality, but can't improve symptoms and quality of life[4]. 
Sodium-glucose co-transporter 2 (SGLT2) inhibitor is a type of drugs used for the treatment of diabetes mellitus. Many large clinical trials conducted in patients at high cardiovascular risks demonstrated to reduce the risk of hospitalization for patients with heart failure[5-7]. However, all the patients included in these trials have diabetes mellitus, and most of them didn't have heart failure at baseline. Thus, many trials have been conducted to explore whether SGLT2 inhibitor could be beneficial for patients with established heart failure, neglect of patients with or without diabetes mellitus.

The object of the present meta-analysis was to pool data from all the large scale, randomized and placebo-controlled trials of SGLT2 inhibitor to obtain more reliable estimates of the efficacy and safety outcomes in patients with heart failure overall.

\section{Methods}

The Preferred Reporting Items for Systematic Reviews and Meta-Analyses

(PRISMA) statement was applied in the current meta-analysis [8].

\section{Data sources and literature search}

Four databases (PubMed, Embase, Cochrane Library, and Web of Science) were searched until March 2020. A comprehensive search was performed to identify published articles of studies on the efficacy and safety of SGLT2 inhibitor in the patients with heart failure neglect with or without diabetes mellitus (hospitalization for heart failure, all-cause death, serious adverse event (SAE), and volume depletion). The search terms were as follows: "SGLT2 inhibitor, heart failure, and SGLT2 inhibitor*". No language limitations were applied.

\section{Study Selection and Inclusion Criteria}

Two reviewers (Xiaofei Wang and Peipei Ge) screened all articles independently, according to the inclusion criteria. Articles like review, abstract or summary presented at the meeting would be excluded. Controversies about studies would be resolved by discussion.

Inclusion criteria were as follows: (1) Full-text and relevant data can be acquired; (2) randomized, placebo-controlled clinical trials involved SGLT2 inhibitor; (3) comparing efficacy and safety of SGLT2 inhibitor in the patients with heart failure. (4) Efficacy outcomes of interest included hospitalization for heart failure and all-cause death. Safety outcomes of interest included SAE (i.e., volume depletion, renal events, major hypoglycemic events, bone fractures, diabetic ketoacidosis, and amputations) and volume depletion.

\section{Quality Assessment and Data Extraction}

Data of study characteristics such as sample size, follow-up period, age and sex were independently extracted by the two reviewers (Xiaofei Wang and Peipei Ge) according to the predefined protocol. The methodological quality of the included trials was assessed using the Cochrane tool for assessing 
randomized clinical trials (RCT). Studies were evaluated according to random sequence generation, allocation concealment, blind of outcome assessment, incomplete outcome data and other bias.

\section{Statistical Analysis}

Stata (Version 14.0. College Station, TX, USA) was used to perform the primary statistical analyses. Data are presented as a mean difference and pooled using the inverse variance for continuous outcome measures. For dichotomous outcomes, data are presented as risk ratios (RR) and pooled using MantelHaenszel random effects model. The $95 \%$ confidence interval $(\mathrm{Cl})$ is used respectively. Heterogeneity was quantified using $\mathrm{I}^{2}$.

Fixed or random effect models were applied to assess the study depending on the heterogeneity $\left(1^{2}\right) .1^{2}$ values of $25 \%, 50 \%$, and $75 \%$ indicated low,

moderate and high degrees of heterogeneity, respectively. If pooled data of analysis showed $\mathrm{I}^{2} \square 50 \%$, the study was homogeneous, and fixed-effect model was used in the study. A random effect model would be used for results when the $\mathrm{I}^{2} \geq 50 \%$. The result was considered to be statistically significant when $p$-value 冈0.05.

\section{Results}

\section{Search results}

Selection of studies for the meta-analysis was showed in Fig. 1. Based on the prespecified search strategy, 1141 potentially relevant articles were searched from the Cocharane library, Embase, PubMed, Web of Science database. Finally, 5 studies[9-13] (involving 8775 patients) met the eligibility criteria. For all 5 trials that assessed the efficacy and safety of SGLT2 inhibitor in patients with heart failure, the results for all-cause death and hospitalization were reported in all trials, and serious adverse event (SAE) and volume depletion were reported in 4 trials $[9-11,13]$.

\section{Characteristics of the studies included and Quality assessment}

Table 1 showed the characteristics of the eligibility studies, and Table 2 showed the baseline characteristics of the patients included in the trials. The mean age was 64.9 years and $29.8 \%$ were women. A total of $3930(44.8 \%)$ patients were known to have diabetes mellitus. All the trials included are randomized, placebo controlled clinical trials. Figure 2 showed the result for quality assessment of trials. 1 trial[12] was at an unclear risk of incomplete outcome data, and 1 trial[13] didn't report the details about binding of outcome assessment (Supplementary material). 
Table 1

Characteristics of eligibility studies

\begin{tabular}{|lllllll|}
\hline Study & $\begin{array}{l}\text { Sample } \\
\text { Size }\end{array}$ & HFrEF & $\begin{array}{l}\text { T2DM } \\
\text { (\%) }\end{array}$ & Comparator & $\begin{array}{l}\text { SGLT2 } \\
\text { inhibitor }\end{array}$ & $\begin{array}{l}\text { Follow- } \\
\text { up }\end{array}$ \\
\hline Rådholm 2018 & 1461 & $+/-$ & 100 & Placebo & Canagliflozin & 3.6 year \\
\hline Kosiborod 2017 & 320 & $+/-$ & 100 & Placebo & Dapagliflozin & 1 year \\
\hline Kato 2019 & 1987 & $+/-$ & 100 & Placebo & Dapagliflozin & 4.2 year \\
\hline $\begin{array}{l}\text { DEFINE-HF } \\
\text { 2019 }\end{array}$ & 263 & + & 62 & Placebo & Dapagliflozin & 3months \\
\hline DAPA-HF 2019 & 4744 & + & 41.8 & Placebo & Dapagliflozin & 1.6 year \\
\hline
\end{tabular}

Table 2

Baseline characteristics of included studies.

\begin{tabular}{|c|c|c|c|c|c|}
\hline Study & $\begin{array}{l}\text { Mean Age, } \\
\text { years }\end{array}$ & $\begin{array}{l}\text { Female, } \\
\mathrm{n}(\%)\end{array}$ & BMI & $E F, \%$ & $\begin{array}{l}\text { Mean GFR } \mathrm{mL} / \mathrm{min} \text { per } \\
1.73 \mathrm{~m}^{2}\end{array}$ \\
\hline $\begin{array}{l}\text { Rådholm } \\
2018\end{array}$ & $63.8 \pm 8.3$ & $648(44.4 \%)$ & $\begin{array}{l}33.2 \pm \\
5.9\end{array}$ & NA & $73.0 \pm 19.6$ \\
\hline $\begin{array}{l}\text { Kosiborod } \\
2017\end{array}$ & $64.2 \pm 7.42$ & $119(37.2 \%)$ & $\begin{array}{l}34.19 \pm \\
5.65\end{array}$ & NA & $70.29 \pm 19.6$ \\
\hline Kato 2019 & $64.3 \pm 6.99$ & $669(33.7 \%)$ & $32.59 \pm 6$ & $\begin{array}{l}49.26 \pm \\
11.28\end{array}$ & $85 \pm 20.08$ \\
\hline $\begin{array}{l}\text { DEFINE-HF } \\
2019\end{array}$ & $61.3 \pm 11.53$ & $70(26.6 \%)$ & $\begin{array}{l}30.65 \pm \\
6.43\end{array}$ & $\begin{array}{l}26.45 \pm \\
8.12\end{array}$ & $69.06 \pm 22.19$ \\
\hline $\begin{array}{l}\text { DAPA-HF } \\
2019\end{array}$ & $66.35 \pm 10.9$ & $1109(23.4 \%)$ & $\begin{array}{l}28.2 \pm \\
5.95\end{array}$ & $\begin{array}{l}31.05 \pm \\
6.80\end{array}$ & $65.75 \pm 19.45$ \\
\hline
\end{tabular}

\section{Efficacy Outcomes}

\section{Hospitalization for heart failure}

All the five trials reported the data of hospitalization for heart failure. The result favored the SGLT2 inhibitor group (RR $0.692 ; 95 \% \mathrm{Cl}, 0.611-0.784 \mathrm{P} \otimes 0.001)$. The heterogeneity is moderate $(12=39.3 \%)$. This indicated that patients who received SGLT2 inhibitors therapy could decrease the rate of hospitalization for heart failure (Figure 2). 
The result of death from any cause was systemically reported in five studies. Patients with heart failure in SGLT2 inhibitor group had a low incidence of death from any cause (RR $0.824 ; 95 \% \mathrm{Cl}, 0.736-0.922$ $P=0.001)$. The heterogeneity among these trials was very low $(I 2=0.0 \%)$ (Figure 3$)$.

\section{Safety Outcomes}

\section{Serious Adverse Events (SAE)}

Data of SAE was included in 4 trials. The incidence of SAE in patients with a treatment of SGLT2 inhibitors was low (RR $0.869 ; 95 \% \mathrm{Cl}, 0.779-0.970 \mathrm{P}=0.012)$. The heterogeneity in these trials was moderate (58.3\%) (Figure4). Because of the moderate heterogeneity in these trials results, we conducted a meta-regression analysis for SAE according to different study characteristics. There was no significant

difference between any of the tested characteristics. A sensitive analysis of SAE was also performed, and the removal of any study did not affect confidence interval significantly.

\section{Volume Depletion}

Four trials reported the data of volume depletion. The incidence of volume depletion between the SGLT2 inhibitors and placebo didn't have a significant difference (RR1.165, 95\% $\mathrm{Cl}, 0.977-1.390 \mathrm{P}=0.089)$. The heterogeneity was very low $\left(I^{2}=0.0 \%\right)$ (Figure5).

Finally, the potential publication bias was tested. Publication bias was not suggested by the funnel plot, Egger's test for all the efficacy and safety outcomes (Figure6).

\section{Discussion}

This study is the first systemic review and meta-analysis to systemically evaluate the efficacy and safety of SGLT-2 inhibitor in patients with heart failure. In our meta-analysis of randomized clinical studies, SGLT-2 inhibitor could significantly decrease the incidence of hospitalization for heart failure and all cause death in patients with heart failure. Compared with placebo, SGLT-2 inhibitors could significantly decrease risk for SAE, and didn't increase occurrence of volume depletion.

Most patients with heart failure suffered from a poor quality of life and high incidence of event rates. Despite advances in the drugs and device for the treatment of heart failure, these population still suffer from symptomatic progression and poor quality of life. Many large clinical studies had indicated that SGLT2 inhibitors had significant reduction in the risk of hospitalization for heart failure in patients with diabetes mellitus $[5-7,14]$. All the patients included in these trials were with diabetes mellitus, and most of them didn't suffer from heart failure at baseline. Whether the heart failure patients without diabetes mellitus could also benefit from the treatment with an SGLT2 inhibitor has not been established. Recently, many large clinical trials has been designed and performed to evaluate the efficacy and safety of SGLT2 
inhibitor in heart failure patients with or without diabetes mellitus, and some had been finished[10, 1519]. We performed the meta-analysis firstly based on the results of these large clinical trials.

For the efficacy outcomes, we observed that SGLT2 inhibitor decreased incidence of hospitalization and all cause death for heart failure as compared with placebo in our meta-analysis. Although many exploratory research has been done, the exact mechanism of the efficacy of SGLT2 inhibitor remain unclear[20]. It is confirmed that patients with heart failure could have a net benefit from SGLT2 inhibitor treatment other than glucose lowering. Thus, our results could further demonstrate the findings of many studies on this subject [21-23].

Our study demonstrated an overall safety profile of SGT2 inhibitors comparable to that of placebo, and the incidence of SAE was similar in both groups. For the reason that SGLT2 inhibitor increases the urinary excretion of glucose and sodium by blocking glucose reclamation in the proximal renal tubules and many patients with heart failure used diuretics at baseline, many experts had potential concerns with volume depletion. However, in our meta-analysis of all these trials, no significant increase was observed in SGLT2 inhibitor group, compared with the placebo.

Our study suggests that SGLT2 inhibitor should be considered in patients with heart failure regardless of presence of diabetes mellitus, given that they safely reduce hospitalization for heart failure and all-cause death in these patients. In future, SGLT2 might be the first choice of hypoglycemic drugs for patients with diabetes mellitus, which increased risk of heart failure and renal disease [24,25]. Ongoing trials in patients with heart failure will clarify the exact mechanism of the salutary effects of SGLT2 in patients with heart failure in future.

\section{Limitations}

We acknowledge that our study has some limitations. The length of follow-up reported in every study was different from each other, which could be attributable to biases. Besides the type and dose of SGLT2 inhibitor in all trials are not consistent either. We observed a moderate heterogeneity in the results of hospitalization for heart failure and SAE, but both meta regression analysis and sensitive analysis didn't identify the exact bias. However, we think the final result wouldn't be greatly affected. More evidence from cohort studies and large clinical trials on the efficacy and safety of SGLT2 inhibitor is thus desirable.

\section{Conclusion}

Our results confirm that SGLT2 inhibitor has salutary effects for patients with heart failure regardless of presence of diabetes mellitus. It will not increase the incidence of SAE either. Compared with placebo, it will not increase the risk of volume depletion.

\section{Abbreviations}

SGLT2, Sodium-glucose co-transporter 2. 
PRISMA, Preferred Reporting Items for Systematic Reviews and Meta-Analyses.

SAE, Serious adverse event.

i.e., id es.

RCT, randomized clinical trials.

RR, Risk ratios.

$\mathrm{Cl}$, Confidence interval.

\section{Declarations}

\section{Ethics approval and consent to participate}

Not applicable.

\section{Consent for publication}

Not applicable.

\section{Availability of data and materials}

All data generated or analyzed during this study are included in this published article.

\section{Competing interest}

The authors declare that they have no competing interests.

\section{Funding}

Not applicable.

\section{Author Contributions}

LXX and ZQ conceived and designed the study. WXF, GP, ZQ searched articles and screened the data. GP, LXX and HAZ performed the statistical analysis. LXX and ZQ wrote the paper. ZQ, WXF, GP, HAZ and LXX reviewed and edited the manuscript. All authors read and approved the manuscript.

\section{Acknowledgements}

Not applicable.

\section{References}


1. Go, A.S., et al., Heart disease and stroke statistics--2013 update: a report from the American Heart Association. Circulation, 2013. 127(1): p. e6-e245.

2. Bui, A.L., T.B. Horwich, and G.C. Fonarow, Epidemiology and risk profile of heart failure. Nat Rev Cardiol, 2011. 8(1): p. 30-41.

3. McMurray, J.J., et al., Angiotensin-neprilysin inhibition versus enalapril in heart failure. N Engl J Med, 2014. 371(11): p. 993-1004.

4. Reddy, P. and A.B. Dunn, The effect of beta-blockers on health-related quality of life in patients with heart failure. Pharmacotherapy, 2000. 20(6): p. 679-89.

5. Guthrie, R., Canagliflozin and cardiovascular and renal events in type 2 diabetes. Postgrad Med, 2018. 130(2): p. 149-153.

6. Wiviott, S.D., I. Raz, and M.S. Sabatine, Dapagliflozin and Cardiovascular Outcomes in Type 2 Diabetes. Reply. N Engl J Med, 2019. 380(19): p. 1881-1882.

7. Rosenstein, R. and A. Hough, Empagliflozin, Cardiovascular Outcomes, and Mortality in Type 2 Diabetes. N Engl J Med, 2016. 374(11): p. 1093-4.

8. Liberati, A., et al., The PRISMA statement for reporting systematic reviews and meta-analyses of studies that evaluate health care interventions: explanation and elaboration. Ann Intern Med, 2009. 151(4): p. W65-94.

9. Radholm, K., et al., Canagliflozin and Heart Failure in Type 2 Diabetes Mellitus. Circulation, 2018. 138(5): p. 458-468.

10. Jhund, P.S., et al., Dapagliflozin Effects on Biomarkers, Symptoms, and Functional Status in Patients With Heart Failure With Reduced Ejection Fraction: The DEFINE-HF Trial. N Engl J Med, 2019. 140(18): p. 1463-1476.

11. McMurray, J.J.V., et al., Dapagliflozin in Patients with Heart Failure and Reduced Ejection Fraction. Clin Pharmacol, 2019. 381(21): p. 1995-2008.

12. Kato, E.T., et al., Effect of Dapagliflozin on Heart Failure and Mortality in Type 2 Diabetes Mellitus. Circulation, 2019. 139(22): p. 2528-2536.

13. Kosiborod, M., et al., Efficacy and safety of dapagliflozin in patients with type 2 diabetes and concomitant heart failure. J Diabetes Complications, 2017. 31(7): p. 1215-1221.

14. Perkovic, V., et al., Canagliflozin and Renal Outcomes in Type 2 Diabetes and Nephropathy. N Engl J Med, 2019. 380(24): p. 2295-2306.

15. Wang, L.F., et al., Randomized, double-blind, placebo-controlled, multicentre pilot study on the effects of empagliflozin on clinical outcomes in patients with acute decompensated heart failure (EMPARESPONSE-AHF). Cardiovasc Diabetol, 2020.

16. Jensen, J., et al., Empagliflozin in heart failure patients with reduced ejection fraction: a randomized clinical trial (Empire HF). Trials, 2019. 20(1): p. 374.

17. Boulton, D.W., et al., Effects of empagliflozin on risk for cardiovascular death and heart failure hospitalization across the spectrum of heart failure risk in the EMPA-REG OUTCOME(R) trial. 
Diabetes Obes Metab, 2018. 39(5): p. 363-370.

18. McMurray, J.J.V., et al., The Dapagliflozin And Prevention of Adverse-outcomes in Heart Failure (DAPA-HF) trial: baseline characteristics. European Journal Of Heart Failure, 2019. 21(11): p. 14021411.

19. Packer, M., et al., Evaluation of the effect of sodiumâ $\epsilon^{\prime \prime}$ glucose co-transporter 2 inhibition with empagliflozin on morbidity and mortality of patients with chronic heart failure and a reduced ejection fraction: rationale for and design of the EMPEROR-Reduced trial. European journal of heart failure, 2019.

20. Zelniker, T.A. and E. Braunwald, Cardiac and Renal Effects of Sodium-Glucose Co-Transporter 2 Inhibitors in Diabetes: JACC State-of-the-Art Review. J Am Coll Cardiol, 2018. 72(15): p. 1845-1855.

21. Bonnet, F. and A.J. Scheen, Effects of SGLT2 inhibitors on systemic and tissue low-grade inflammation: The potential contribution to diabetes complications and cardiovascular disease. Diabetes Metab, 2018. 44(6): p. 457-464.

22. Inzucchi, S.E., et al., Improvement in Cardiovascular Outcomes With Empagliflozin Is Independent of Glycemic Control. Circulation, 2018. 138(17): p. 1904-1907.

23. Zelniker, T.A., et al., SGLT2 inhibitors for primary and secondary prevention of cardiovascular and renal outcomes in type 2 diabetes: a systematic review and meta-analysis of cardiovascular outcome trials. Lancet, 2019. 393(10166): p. 31-39.

24. Cavender, M.A., et al., Impact of Diabetes Mellitus on Hospitalization for Heart Failure, Cardiovascular Events, and Death: Outcomes at 4 Years From the Reduction of Atherothrombosis for Continued Health (REACH) Registry. Circulation, 2015. 132(10): p. 923-31.

25. Martinez-Ceron, E. and F. Garcia-Rio, Mortality and Cardiovascular Disease in Type 1 and Type 2 Diabetes. N Engl J Med, 2017. 377(3): p. 300.

\section{Figures}




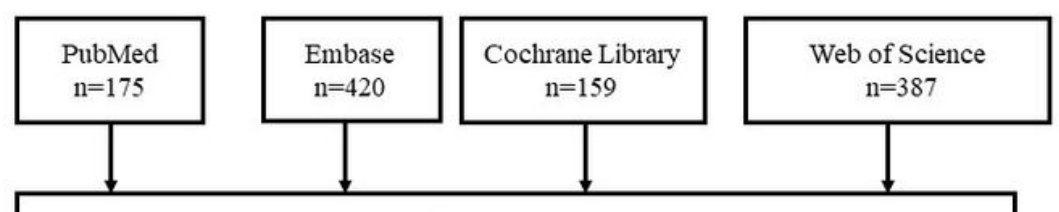

1141 potentially relevant articles identified through electronic databases

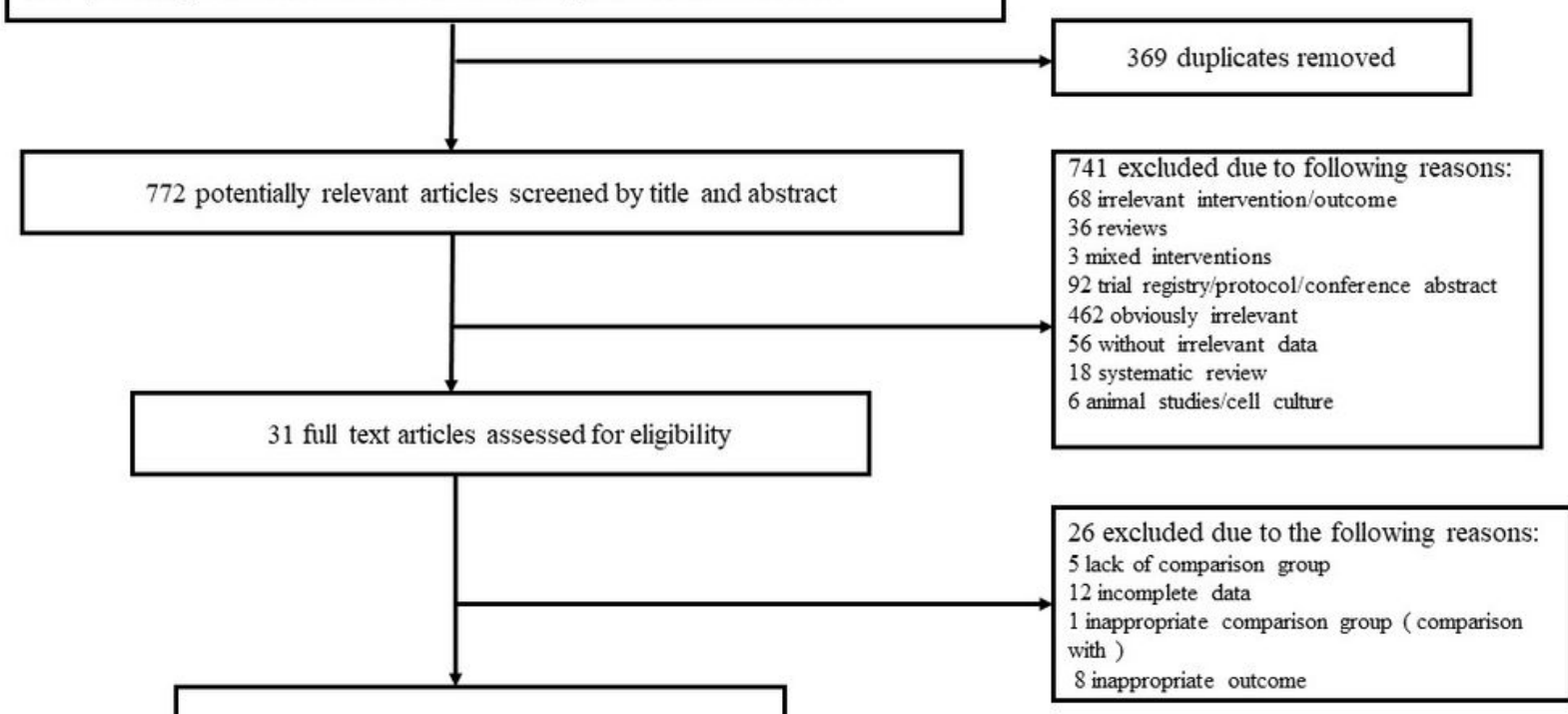

5 articles included in meta-analysis

\section{Figure 1}

Selection of studies for the meta-analysis 
ID

RR (95\% Cl)

Weight

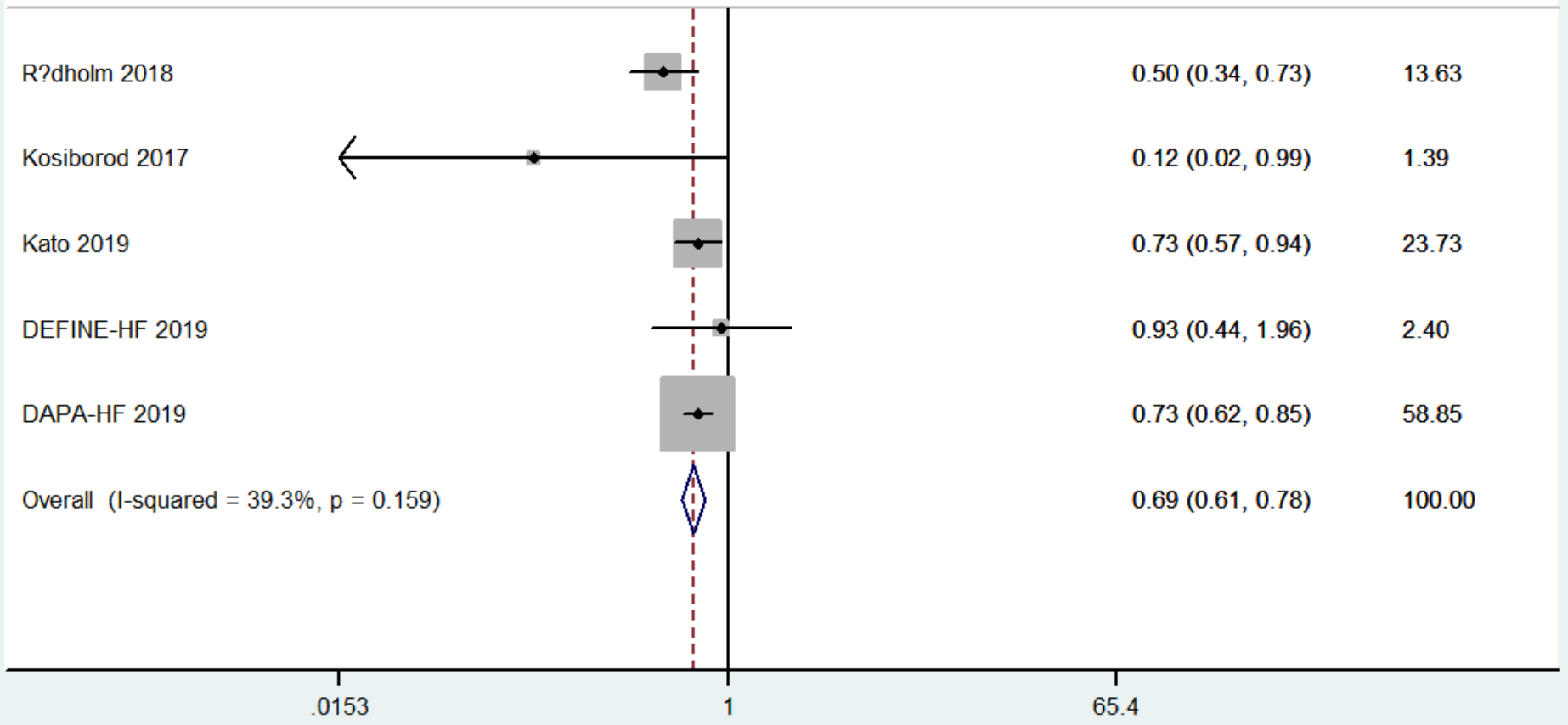

Figure 2

Result for quality assessment of trials. 


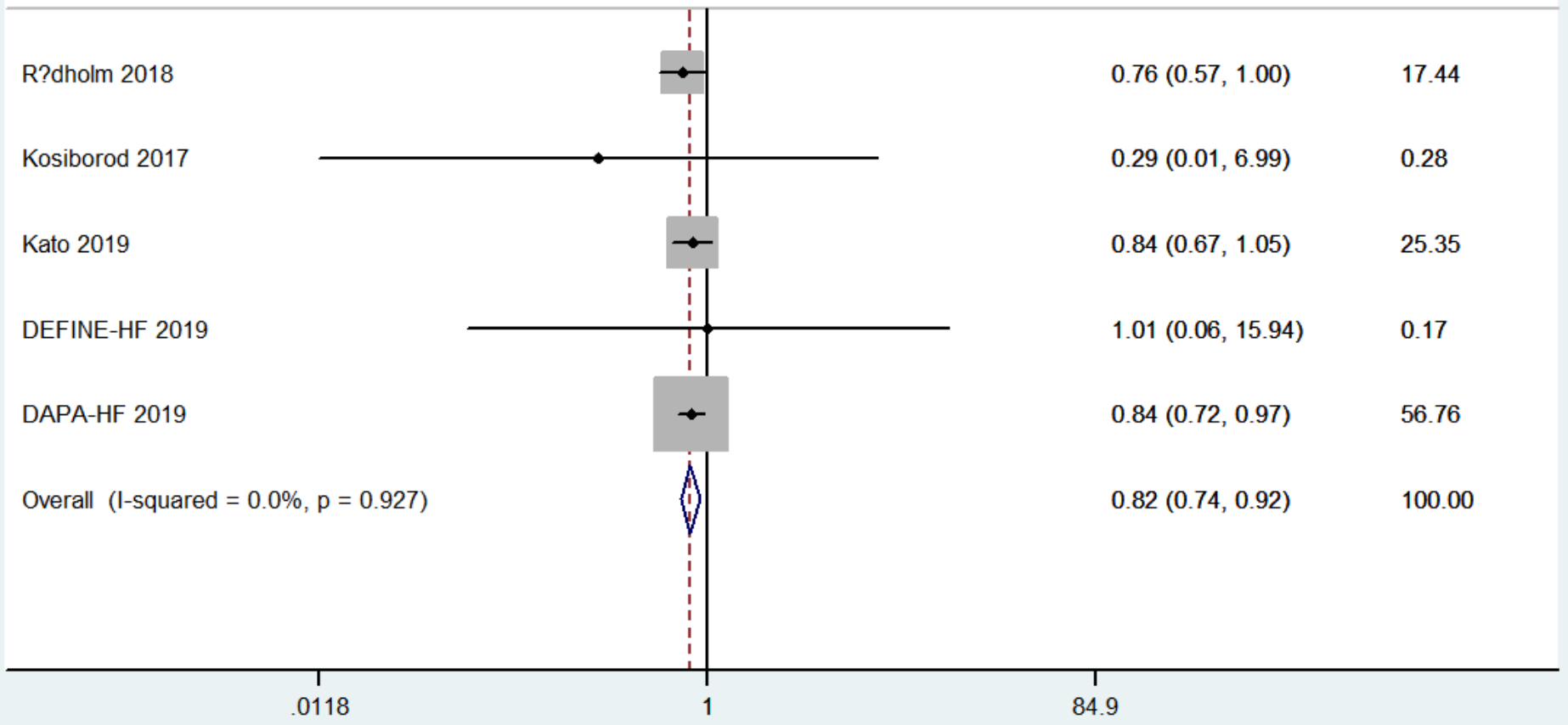

\section{Figure 3}

The result of death from any cause was systemically reported in five studies. 


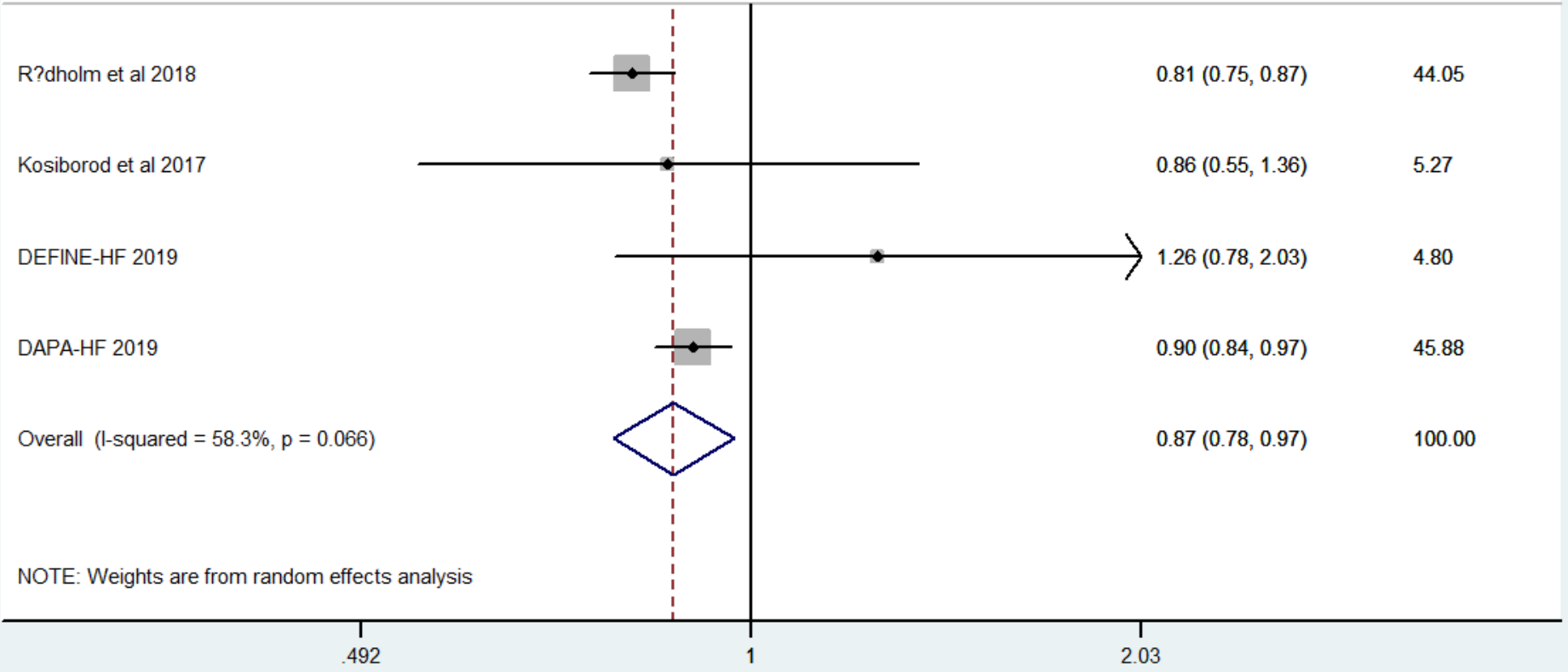

\section{Figure 4}

Serious Adverse Events (SAE) 


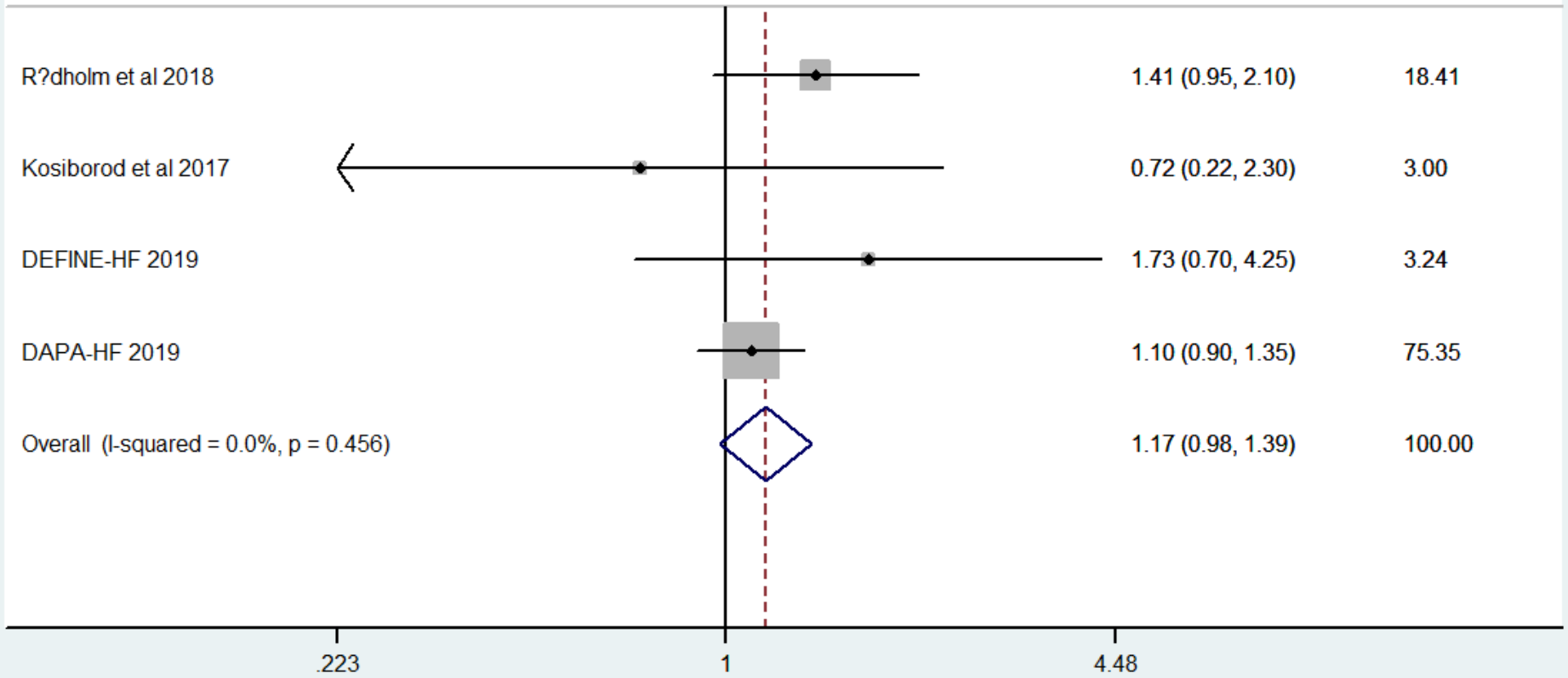

\section{Figure 5}

\section{Volume Depletion}



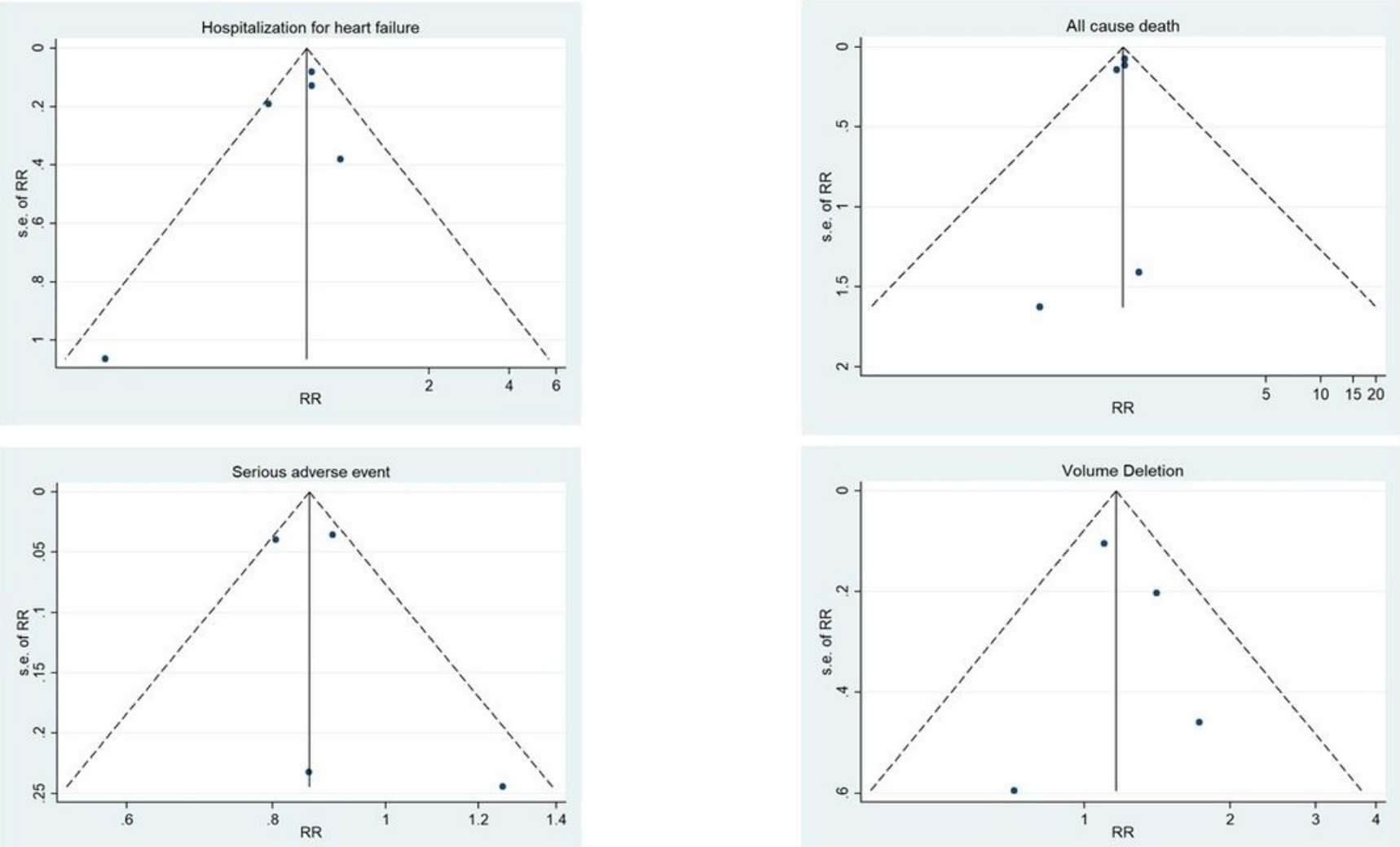

Figure 6

Potential Publication Bias

\section{Supplementary Files}

This is a list of supplementary files associated with this preprint. Click to download.

- Riskofbiassummary.jpg

- Riskofbiasgraph.jpg 